\title{
Nested Case-control Study
}

National Cancer Institute

\section{Source}

National Cancer Institute. Nested Case-control Study. NCI Thesaurus. Code C48202.

A study in which a group or cohort of individuals is followed for a certain length of time or until a specific outcome is reached. Studies of this type incorporate the strengths of both cohort and case-control studies but eliminates a portion of the methodologic challenges inherent in both types of studies. 